\title{
Die Erzählfähigkeit eines zweisprachig aufwachsenden Geschwisterpaares: eine exemplarische Analyse
}

\begin{abstract}
This paper describes the ability of German-Portuguese bilingual siblings to narrate in German. The paper deals with the underlying theory of 'Functional Pragmatics' and describes within this theory the complex verbal pattern of narration in everyday life. In order to do this, a selected corpus will be analysed focussing on the conditions and characteristics of narration.
\end{abstract}

Keywords: Bilingualism; Functional Pragmatic; Narration; German as a Foreign Language; Early Language Promotion.

Resumo: Este artigo tem como objetivo analisar a capacidade de crianças narrarem em alemão. Como exemplo, observaram-se dois irmãos bilíngües (alemão - português). Na primeira parte, será explicada a teoria da 'Pragmática Funcional', assim como o padrão acional verbal complexo da narração na vida cotidiana. A segunda parte, empírica, explicará as condições e características de uma narração através de um corpus selecionado.

Palavras-chave: Bilingüismo; Pragmática funcional; Narração; Alemão como língua estrangeira; Fomento da linguagem em fase pré-escolar.

Die Autorin war 2005/2006 DAAD-Sprachassistentin an der Universidade Estadual de Campinas (UNICAMP). 
Stichwörter: Zweisprachigkeit; Funktionale Pragmatik; Erzählung; Deutsch als Fremdsprache; frühkindliche Sprachförderung.

\section{Einleitung}

Ziel dieser Arbeit ist es, exemplarisch die Fähigkeit des freien Erzählens im Deutschen an einem bilingual aufwachsenden Geschwisterpaar im Alter von sechs und acht Jahren zu analysieren. Im ersten Teil der Arbeit wird die Diskursart Erzählen als ein komplexes Handlungsmuster im Sinne der Funktionalen Pragmatik beschrieben. Darauf folgend werden die Bedingungen bzw. die Strukturmerkmale für ein „erfolgreiches” Erzählen erklärt. Die eigentliche Beschreibung und Interpretation des Auswahlkorpus hinsichtlich der Analyse der Erzähleinheiten erfolgt im zweiten, empirischen Teil, dem sich eine Bewertung und Schlussbetrachtung anschließt. Außerdem sollen im Laufe der Analyse noch folgende Fragen beantwortet werden: Welche Motive verfolgen die Kinder beim Erzählen? Wen wählen sie als Zuhörer? Wie beteiligen sie sich als Zuhörer? Welche anderen sprachlichen Handlungsmuster werden realisiert (s. Meng et al. 1991)?

\section{A) Theoretischer Teil}

\section{A-1. Die Theorie der Funktionalen Pragmatik}

Die Basis dieser Arbeit bildet die Theorie der Funktionalen Pragmatik.

Das sprachliche Handeln ist gesellschaftliche Tätigkeit gesellschaftlicher Subjekte. Es bedient sich gesellschaftlich ausgearbeiteter Formen und verläuft in ihnen. Das sprachliche Handeln ist Teil sonstigen menschlichen Handelns und in es eingebunden. Es partizipiert also an dessen allgemeiner Charakteristik und Vielfalt (EHLich/ReHbeIN 1979: 243).

Die Auffassung von Sprache in der funktional-pragmatischen Kommunikationsanalyse betrachtet Sprache als eine Möglichkeit des gesellschaftlichsozialen Handelns. Sie wird durch Interaktion der Aktanten erworben als 
auch entwickelt und wird als „etwas Funktionales behandelt” (EHLICH 1999: 6), in deren Mittelpunkt Zwecke stehen. Die Zwecke sind allerdings nicht beliebig, sondern je nach Bedürfnis bildet sich ein bestimmter Zweck heraus (EHLich \& REHBEIN 1979). Schon die frühen Schreie des Kindes dienen als Ausdrucksmittel für Zustände und sollen auf seine Bedürfnisse wie z.B. Hunger aufmerksam machen (BüHLER 1922). Durch Aneignung sprachlicher Mittel, die das Kind aufgrund von Interaktion mit der Umgebung erwirbt, lernt es, seine Bedürfnisse differenzierter auszudrücken.

Interaktionen erfolgen in sprachlichen Handlungen. Ihnen liegen immer sprachliche Handlungsmuster zugrunde. EHLICH \& REHBEIN (1979: 250) sprechen von „standardisierten Handlungsmöglichkeiten”. Die einzelnen Muster können dabei einfach (z.B. Fragen) oder komplex sein, d.h. aus mehreren einfachen Sprechhandlungen (z.B. Erzählungen, Beschreibungen) zusammengesetzt sein. Eine einzelne Sprechhandlung wiederum besteht aus dem Vollzug dreier Akte: dem Äußerungsakt, dem propositionalen Akt und dem illokutiven Akt. Der Äußerungsakt ist für die lautliche, morphologische und grammatikalische Anordnung der Wörter 'zuständig'. Der propositionale Akt soll den Informationsgehalt vermitteln, während der illokutive Akt die spezifische kommunikative Rolle der Äußerung bestimmt. Den einzelnen Akten werden als weitere sprachliche Kategorie die Prozeduren untergeordnet (s. EHLICH 1999). Sie unterstützen die mentale Verarbeitung des Sprechers und sind die 'feineren' sprachlichen Ausdrücke, sozusagen die kleinsten Einheiten. Hier sind vor allem die operativen (z.B. Konjunktionen) und die deiktischen ${ }^{1}$ Prozeduren (z.B. Demonstrativ-, Personal- und Possessivpronomen) zu nennen.

Zusammenfassend lässt sich sagen, dass es nicht ausreicht, sprachliche Äußerungen isoliert in phonologischer, morphologischer, semantischer oder syntaktischer Hinsicht zu betrachten (WEBER 1982). Vielmehr müssen Form (Art des Handlungsmusters, der Akte und der Prozeduren) und Funktion (Zweck) in ihrer wechselseitigen Beeinflussung betrachtet werden. Weiterhin

1 Deiktisch kommt von Deixis (altgriechisch) = Zeigen, d.h. die Bedeutung des Wortes erschließt sich aus der Situation. 
ist für die sinnvolle Interpretation der Äußerung auf pragmatischer Ebene das situative Umfeld zu beachten (a.a.O.). Denn die Situation entscheidet über die Anwendung der jeweiligen Muster. Daher müssen auch nonverbale Elemente sowie soziale Faktoren bei der Analyse miteinbezogen werden.

Kinder stehen also vor der Aufgabe, sich im Laufe ihres Spracherwerbsprozesses die lautliche Produktion, die grammatikalisch richtige Anordnung und die semantische Bedeutung von Sprache anzueignen, sowie deren Handlungsformen. Zweisprachig aufwachsende Kinder stehen noch dazu vor der Schwierigkeit, dass sie sich das Wissen über sprachliche Handlungen in zwei Sprachen aneignen müssen. Zudem müssen sie über ein größeres sprachliches Repertoire verfügen als monolingual aufwachsende Kinder, damit sie die Handlungsmuster sowohl in der Erst- als auch in der Zweitsprache angemessen durchführen können.

\section{A-1.1. Das Transkribierverfahren}

Die Funktionale Pragmatik untersucht Sprache in ihrer gesellschaftlichen Wirklichkeit, d.h. ihr Gegenstand ist nicht eine ausgezeichnete Sprachform wie z.B. die Hochsprache (EHLICH 1983b). Ihre Untersuchungen basieren daher auf Empirie, um dem Grundsatz der Authentizität gerecht $\mathrm{zu}$ werden. Da mündliche Kommunikation flüchtig ist, muss sie deshalb (vor allem für die wissenschaftliche Untersuchung) aufgezeichnet und schriftlich umgesetzt werden, d.h. transkribiert werden (a.a.O.). EHLich \& REHBEIN (1976) haben dazu ein Verfahren entwickelt; die halbinterpretative Arbeitstranskription (HIAT). Ziel dieser Transkription ist es, mündliche Kommunikation so genau wie möglich wiederzugeben. Zu den wichtigsten Verfahren von HIAT gehört daher die Partiturschreibung (veranschaulicht durch Flächenschreibung). Sie gibt das gleichzeitige Gespräch von mehreren Personen wieder und verdeutlicht die Synchronität der Sprecher. Die literarische Umschrift, als ein weiteres Verfahren, bringt die Varianten der Hochsprache wie auch dialektale Färbung (z.B. „Sach mal” anstatt „Sag mal!”) und Überlegungspausen der Sprecher zum Ausdruck. Die Verwendung der Satzzeichen sowie die Groß- und Kleinschreibung ist interpretativ. Es liegt im Ermessen des Transkribierenden, ob er eine Äußerung für abgeschlossen hält und dies durch ein Satzzei- 
chen markiert oder eine Markierung unterlässt. Besonders Kinder sprechen oft in Ein- oder Zweiwortsätzen; bei ihrer Interpretation spielt daher die hermeneutische Methode eine große Rolle. Folgende Zeichen werden im Transkript verwendet:
/ Abbruch
((4s)) längere Pause
( ) Akustisch Unverständliches
L, C, T Sprechersiglen
Emphase
(( )) nicht-phonologische Beschreibung
[ ] simultanes Sprechen

Die vorliegenden Aufnahmen wurden nach dem HIAT-Verfahren verschriftlicht. Die Aufnahmen selbst wurden mit einer Panasonic S-VHS-C Kamera NV-88 gemacht.

\section{A-2. Das sprachliche Curriculum der Kinder}

Die Aufnahmen, die dieser Arbeit zugrunde liegen, stammen von einem brasilianischen Geschwisterpaar. Tobias (T), der Ältere, wurde im Februar 1995 noch in Deutschland geboren, aber schon vier Monate nach seiner Geburt zogen die Eltern (wieder) nach Brasilien. Dort wurde das zweite Kind, Claudia (C), geboren. Die Mutter ist Brasilianerin. Sie lebte schon acht Jahre in Deutschland, wo sie auch ihren Ehemann, einen Deutschbrasilianer kennen lernte. Er wuchs ebenfalls in Brasilien auf, war aber von Geburt an der deutschen und der portugiesischen Sprache 'ausgesetzt', wobei die Umgebungssprache portugiesisch war und in der Familie beide Sprachen gemischt wurden. Dies entspricht, laut Romaine (1989: 166 ff.) in ihrer Typologisierung, dem simultanen Bilingualismuserwerb Typ 6:

\footnotetext{
'Mixed Languages' (Sprachenmischung)

Parents: The parents are bilingual.

Community: Sectors of community may also be bilingual.

Strategy: Parents code-switch and mix languages.
}

Die Eltern von T und C sprachen miteinander Portugiesisch. Die Deutschkenntnisse der Mutter reichten nicht für eine problemlose Kommunikation im Deutschen aus. Der Eltern-Kind-Diskurs verlief daher in Portugiesisch. 
Außerdem hatten die Kinder in Brasilien keinen nennenswerten Kontakt mit der deutschen Sprache. Bis zum Alter von 6,6 Jahren (Tobias) und 5,3 Jahren (Claudia) lebten die Kinder in Brasilien. Dann zogen die Eltern im August 2001 wieder nach Deutschland. Der Eltern-Kind-Diskurs verlief auch in Deutschland in der portugiesischen Sprache und entspricht laut ROMAINE (a.a.O.: 166 ff.) Typ 3 ihrer Klassifizierung:

'Non dominant home language without a community support'

Parents: The parents share the same native language.

Community: The dominant language is not of that of the parents.

Strategy: The parents speak their own language to the child.

Kurz danach wurde T im Alter von sechs Jahren - mit minimalen Kenntnissen in der deutschen Sprache - eingeschult. Nach zwei Monaten musste er aufgrund mangelnder Deutschkenntnisse die Schule verlassen. Die ganze Zeit über war es ihm nicht möglich, dem Unterricht zu folgen. Von seinen Mitschülern wurde er auch gehänselt. T wurde zurückgestellt, was für ihn nicht ohne psychische Folgen blieb, und kam zusammen mit C wieder in den Kindergarten. Seit Februar 2002 besuchen sie das Projekt KIKUS-Sprachförderung im Kindergarten. An diesem Projekt beteiligen sich mittlerweile neun Kindergärten in München. Einmal in der Woche besuchen die Kinder im Alter von vier bis sechs Jahren Kindersprachkurse von 90 Minuten, die sie in ihrem Deutscherwerb bis zur Einschulung unterstützen sollen, um annähernd die gleichen Voraussetzungen und Chancen bei der Einschulung zu haben wie ihre zukünftigen deutschsprachigen Klassenkameraden. Bereits mit Eintritt in die Schule wird eine gewisse sprachliche und soziale Kompetenz vorausgesetzt. Der KIKUS-Unterricht findet in Kleingruppen (mit nicht mehr als zehn Kindern) am Nachmittag abseits vom Kindergartenalltag statt. Auf spielerische Art und Weise bekommen die Kinder Grammatik sowie sprachliche Handlungsmuster beigebracht und mit Hilfe von Liedern, Spielen und anderen Materialien wird ihr Wortschatz erweitert. Die Kurse werden von Honorarkräften geleitet, die auch Fortbildungen für Erzieherinnen anbieten. Finanziert werden die Kurse durch Elternbeiträge und durch die Evangelische Ausländerarbeit in München. 
Seit dem Besuch des Kindergartens und des KIKUS-Projekts, in denen die institutionelle Sprache das Deutsche ist, fängt das Geschwisterpaar auch an, untereinander Deutsch zu sprechen. Zehn Monate nach ihrer Ankunft in Deutschland sprechen die Kinder nun auch vermehrt Deutsch mit ihrer Mutter, wobei die Mutter selbst noch Schwierigkeiten hat, sich wieder in die deutsche Sprache hineinzufinden. Zusammenfassend kann man sagen, dass die Kinder seit ihrer Geburt die meiste Zeit in einer portugiesischen Sprachumgebung aufgewachsen sind und erst seit knapp einem Jahr intensiven deutschen Sprachkontakt haben. Sie sind gezwungen, sich nicht nur die phonologischen, morphologischen, lexikalischen und syntaktischen Strukturen der Sprache anzueignen, sondern müssen jetzt auch lernen, in der Zweitsprache sprachlich zu handeln und dazu die geeigneten Handlungsmuster zu verinnerlichen - wie zum Beispiel das Erzählen in der Zweitsprache.

\section{A-3. Das sprachliche Handlungsmuster Erzählen}

Erzählen ist als sprachliches Handeln integriert in die sonstigen Handlungsbezüge der gesellschaftlichen Aktanten. Es ist eines der prominentesten Mittel, mit denen der Transfer von Erfahrung bewältigt werden kann (EHLich 1980: 20).

Erzählen ist somit Teil des gesellschaftlichen Handelns und realisiert sich in der Interaktion der Aktanten. Es kann sich im Sinne der funktionalpragmatischen Kommunikationsanalyse unter der Bedingung der Kopräsenz von Sprecher und Hörer abspielen. Bei dieser gleichzeitigen (auch lokalen) Anwesenheit der Aktanten handelt es sich um diskursives Erzählen (auch konversationelles Erzählen genannt). „Wir verstehen konversationelle Erzählung als grundsätzlich mündlich konstituierte Diskurseinheiten, die sich ohne vorherige Festlegung in Gesprächen realisiert" (QuAsthoff 1983: 52). Bei fehlender Kopräsenz, d.h. in einer 'zerdehnten' Sprechsituation (EHLich 1984b: 18) liegt textuelles Erzählen vor. Das typisch diskursive Erzählen findet in der Alltagskommunikation statt, während textuelles Erzählen im literarischen Erzählen vorkommt. Wenn wir an Erzählen denken, verbinden wir es häufig zuerst mit der literarischen Gattung Erzählung (EHLIcH 1980). 
Denn unser Bild vom Erzählen ist geprägt von den Erzählungen professioneller Erzähler, der Literaten, deren Erzählung wir lesen. Dieses Erzählen ist ein schriftliches Erzählen. Das alltägliche Erzählen wird demgegenüber abgedrängt in den Untergrund des ,gewöhnlichen" Alltags (...) (EHLich 1984b: 7).

In der Wissenschaft wurde die Beschäftigung mit den 'alltäglichen' Erzählungen vernachlässigt. Dabei spielen sie im Alltag eine große Rolle. Erzählt wird überall, sei es in der Familie, am Arbeitsplatz, im Unterricht oder auf der Straße. Die Erzählungen sind fiktiv oder real, selbsterlebte Geschehnisse oder die Erlebnisse eines anderen. Die verschiedenen Erzählarten lassen sich eben nicht nur in der großen Literatur wiederfinden, sondern kommen auch in der Alltagskommunikation vor. Das Erzählen ist die am meisten verwendete Form der Darstellung von Ereignissen (BOUEKE et al. 1995). Da die vorliegende Arbeit die Erzählfähigkeit bei Kindern zum Thema hat, interessiert hier der Kommunikationstyp des Alltags, d.h. die nicht-professionelle, mündliche Kommunikation. Nach EHLICH (1983a) erfährt der, der gut erzählt, von seiner Umgebung besondere Achtung. Demjenigen, der schlecht erzählt, wird weniger zugehört, und er wird ausgeschlossen. Wer überhaupt nicht erzählt, begrenzt seine sozialen Handlungsmöglichkeiten. Wer in einem institutionellen Umfeld zu oft erzählt, kann in einen Konflikt geraten. Wie man sieht, kann mangelnde Erzählkompetenz negative Folgen auf das soziale Leben eines Individuums haben. WAgner \& STEINSTRÄTER (1989: 60) formulieren dazu griffig: „Sprechen lernt das Kind, um die Bezugsperson anzumischen. Erzählen lernt das Kind, um in der Familie mitzumischen." In seiner allgemeinen Bedeutung wird von Erzählen in ganz unterschiedlichen Situationen gesprochen, auch wenn z.B. nach der Aufforderung: „Erzähl mal wie es in der Schule war?’ nur eine Aufzählung von Fächern erfolgt (BOUEKE et al. 1995). Der alltägliche Gebrauch des Begriffs Erzählen wird hier nicht nur im Sinne des konversationellen oder literarischen Erzählens gebraucht (z.B. „Lass dir nichts erzählen!'), sondern weist nach EHLICH (1983a: 128) „eine semantische Vielfalt auf."'

2 EHLich (1983A: 128) unterscheidet ein 'erzählen 1' als allgemeiner Oberbegriff und 'erzählen 2' mit den Unterklassen: berichten, mitteilen, schildern usw. 
Wie wir anfangs festgestellt haben, liegt jedem sprachlichen Handlungsmuster ein Zweck zugrunde. Welchen Zweck verfolgt das Erzählen? Wir erzählen über eine unerhörte Begebenheit, weil wir davon ausgehen, dies könnte für den Zuhörer unterhaltend, interessant sein. Oder wir beabsichtigen, ein bestimmtes Bild bzw. Image von uns zu fördern. Das kann auch durch eine „Leidensgeschichte” vermittelt werden (der Erzähler stellt sich als ein ungerecht Behandelter dar). Erzählen wird auch funktionalisiert, um soziale Nähe zu schaffen, oder dient der psychischen Selbstentlastung. Es gibt also viele Typen von Erzählungen. Sie lassen sich als 'Siegesgeschichte', 'Erbauungsgeschichte', 'Leidensgeschichte' oder 'Glücksgeschichte' klassifizieren (s. ReHbein 1980). Der Typ von Erzählung bestimmt dabei die Teilhabe (mitlachen, mitweinen, mithoffen usw.). Zweck des Erzählens ist es, eine erlebte oder erfundene Geschichte so zu präsentieren, dass der Hörer den Ablauf in seiner Vorstellung nachvollziehen und die Bewertung durch den Sprecher teilen kann (ZIFONUN et al. 1997: 23).

Wie sieht der Durchlauf der Diskursart Erzäblen aus? Im Alltag beginnt eine Erzählung häufig mit einer Ankündigung oder einem ,abstract” (Hoffmann 1984: 205) wie z.B. „Gestern ist mir vielleicht was passiert!” oder „Ich muss dir unbedingt erzählen, was ich morgen machen werde!” Mittels der Ankündigung verlangt der Sprecher einerseits die Aufmerksamkeit des Rezipienten und versucht andererseits, ein „,exklusives” (BOUEKE et al. 1995: 15) Rederecht zu erlangen. Nach BouEKE et al. (a.a.O.) lässt sich daran auch erkennen, dass mit der zu erwartenden Geschichte zugleich ein Versprechen verbunden ist, sie muss etwas Besonderes, Außergewöhnliches bieten und einen Handlungszusammenhang mit unerwartetem Verlauf darstellen. „Das Erzählenswerte einer Erzählung liegt im Wesentlichen in der Ungewöhnlichkeit der Geschichte, die erzählt wird" (QuAsthoff 1983: 54). Fehlt diese 'Kategorie der Ungewöhnlichkeit' (a.a.O.), kann dies zu befremdeten Reaktionen oder Unverständnis der Zuhörer führen.

Damit die Erzählung ${ }^{3}$ zustande kommt, muss der Sprecher mental seine Wissensbestände arrangieren, um eine kohärente Ereignisabfolge zu garantieren. Die Wissensbestände werden nach einem handlungsbezogenen

3 Nach Quasthoff (1983) ist eine Erzählung das Resultat des Erzählens. 
Schema angeordnet (Konstellation, Handlungsabfolge, Relevanzpunkt, Abschluss, Bewertung). In einem nächsten Schritt ist es die Aufgabe des Erzählers, die Verstehensvoraussetzungen zu schaffen (Konstellation), um den Zuhörer zu 'orientieren'. „Das Orientieren ist eine fortlaufende Aktivität des Erzählers mit dem Zweck, dem Rezipienten eine referentielle Basis für die Abwicklung von Handlungs- bzw. Ereignisketten im Erzählkern [...] zu geben [...]" (Hoffmann 1984: 205). Der Rezipient (Hörer) sollte nach dieser einleitenden Orientierung einen ausreichenden Verstehenshintergrund haben, um den anschließenden Verlauf der Geschichte nachvollziehen zu können.

Der Handlungsverlauf der Geschichte wird dann normalerweise in der Reihenfolge dargestellt, wie er sich in der Wirklichkeit ereignet hat. Die einzelnen Handlungsschritte werden nach Zifonun et al. (1997) aus der Perspektive des Sprechers wiedergegeben, sodass diese Perspektive auch für den Hörer nachvollziehbar ist. Nachdem die Relevanzpunkte deutlich gemacht wurden, erfolgt die Bewertung. Relevanzpunkte können durch Tempuswechsel, Übergang zur direkten Rede, durch ein Verbum dicendi oder durch explizite Kommentierung markiert werden (ZIFONUN et al. 1997). Laut HofFMANN (1984: 206) gelingt die Erzählung, „wenn der vom Erzähler gesetzte Relevanzpunkt in das Relevanzsystem des Rezipienten übernommen werden kann.”

Für die Diskursart Eræählen kommt jetzt die entscheidende Abschlusshandlung: Das Bewerten. Bei QuAsthoff (1983) bildet diese 'Kategorie der Evaluation' neben der 'Kategorie der Ungewöhnlichkeit' die zweitwichtigste Kategorie. Bei Wagner \& SteInStRÄter (1989) gilt sie als die grundlegende Fähigkeit beim Erzählen. Für das Erzählen von Alltagsgeschichten hat das Bewerten einen doppelten Aspekt. Auf der Seite des Erzählers sorgt die Bewertung für die emotionale Verankerung der Ereignisse als Erlebnis. Durch die Bewertung werden beliebige Vorkommnisse zu 'meiner Geschichte' (a.a.O.: 49). Für den Zuhörer signalisiert die Bewertung, dass ihm eine Geschichte erzählt wird und kein anderes Handlungsmuster vorliegt. Ohne eine Bewertung fragt er sich, was die Geschichte soll, denn eine Geschichte, die keine Bewertung erfährt, ist allenfalls eine Kette von Geschehnissen (a.a.O.).

Im Folgenden werde ich die Sprachaufnahmen von T und C im Hinblick auf ihre Erzählfähigkeit analysieren. Dabei werden die Strukturmerkmale des Erzählens näher betrachtet. 


\section{B) Empirische Analyse der Erzähleinheiten}

\section{B-1. Transkript (segmentierte Form)}

$\mathrm{C}=$ Claudia, $\mathrm{T}=$ Thomas, $\mathrm{L}=$ Lehrerin

(s1) C: Regen.

(s2) T: Regenwu, Regen, Regenwurm.

(s3) L: Regenschirm.

(s4) T: Regen.

(s5) L: Regenschirm.

(s6) T: Regenschirm, Regenschirm ((flüsternd)).

(s7) C: Mütze ( ) ((auf die nächsten Abbildungen zeigend)).

(s8) T: Hüt.

(s9) L: Was ist denn ein Regenwurm?

(s10) T: Hut.

(s11) L: Was ist ein Regenwurm, weißt du das noch?

(s12) T: Portugiesisch?

(s13) L: Nee. Weißt du, was ein Regenwurm ist, weil du eben gesacht hast Regenwurm.

(s14) T: Ja, ich weiß.

(s15) C: Ja. Wie ein Schlange ((macht eine gestische Bewegung dazu)).

(s16) L: Genau.

(s17) T: Ja.

(s18) C: Aber, aber ich hab schon zwei gehabt. Aber eine hat ganz ganz Wasser, ganz Hoch-wasser ((gestikuliert dazu)) und der hat schon tot. Und vom vom Thomas hat sich verlieren.

(s19) L: Was, (weil) der eine ist ertrunken, tot, weil da soviel Wasser da war und von Thomas, den hast du verloren?

(s20) C: Hm.

(s21) L: Hm. Hört sich ja gut an. OK.

(s22) T: Weißt du was, was ich gemacht habe? 


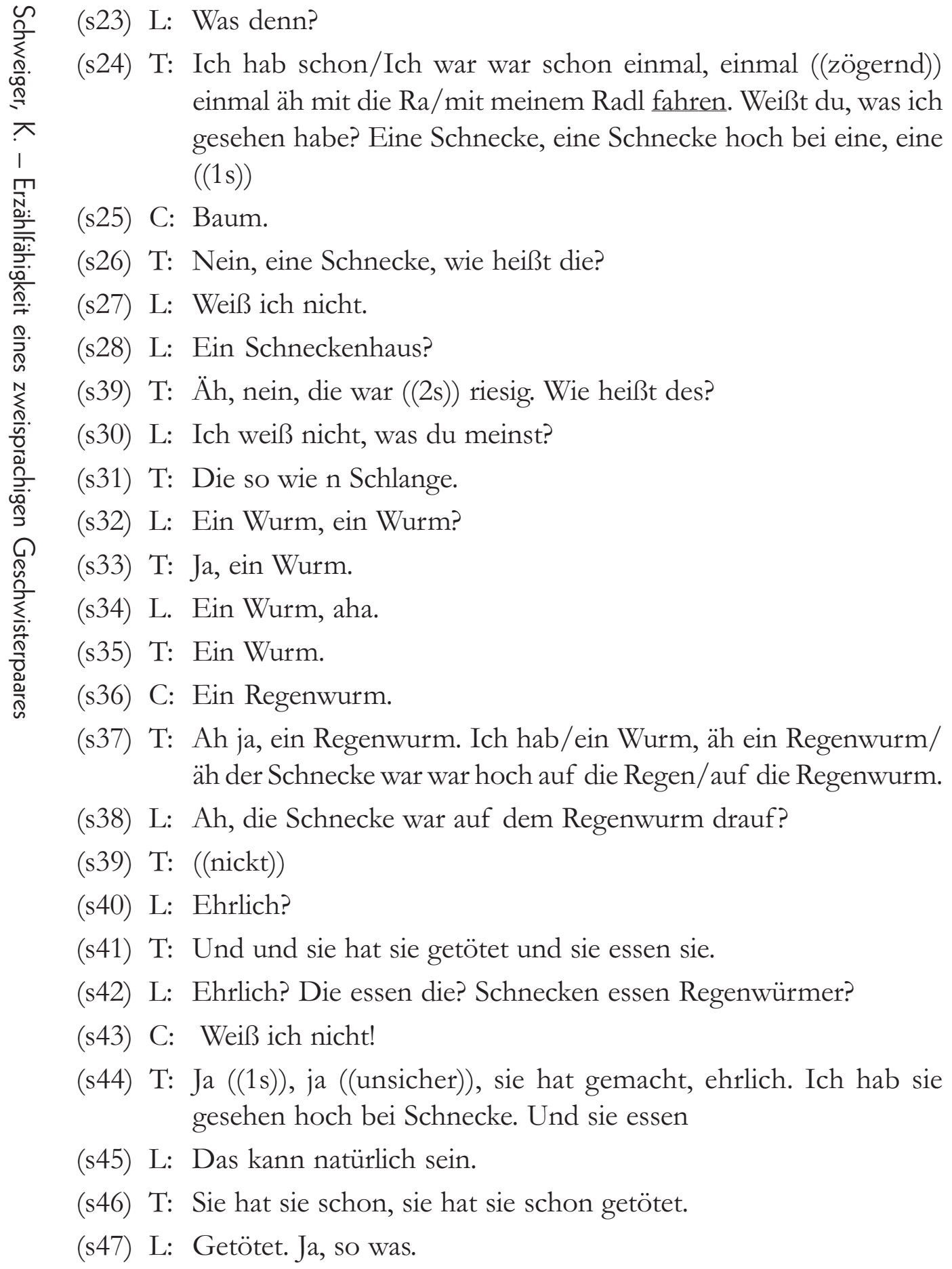




\section{B-2. Allgemeiner Handlungsablauf}

Bei dem vorliegenden Transkriptausschnitt befinden sich die Kinder im Kindergarten. Sie hatten gerade Unterricht am Nachmittag im Rahmen des KIKUS-Projekts. Während sie noch auf ihre Mutter warten, schauen sie mit der Lehrerin (L) ihre Hausaufgabenmappe an. L zeigt auf verschiedene Abbildungen. Die Kinder sollen diese auf Deutsch benennen. Bei einer Abbildung handelt es sich um einen Regenschirm. Initiativ reagiert $C$ und nennt den Ausdruck Regen. Und gibt sich damit zufrieden. T scheint zu wissen, dass es nicht nur Regen ist und versucht den Ausdruck zu finden. Er beginnt mit Regenwu, scheint sich aber unsicher zu sein, ob dies der richtige Ausdruck ist. Schließlich entscheidet er sich nach einem Zögern doch für (s2) Regenwurm. L nennt ihm die richtige Bezeichnung (s3) Regenscbirm. T versucht daraufhin Regenschirm zu imitieren, aber schafft es nur bis (s4) Regen. Erst in einem zweiten Anlauf nach der Verbesserung von L gelingt ihm die vollständige Benennung, die er für sich dann noch einmal leise wiederholt.

Hier liegt ein sprachliches 'Ausprobieren'4 im Äußerungsakt vor. GarLIN (1994) weist darauf hin, dass ein typisches Merkmal dieser Form des Ausprobierens das Fehlen eines illokutiven und propositionalen Gehalts ist. Diesem sprachlichen Muster liegt kein sichtbarer Zweck zugrunde. Trotzdem ist das Ausprobieren in phonologischer Hinsicht Teil des interaktiven sprachlichen Ablaufs, da ihm der Imitationsmechanismus zugrunde liegt (a.a.O.). Das betonte Vorsprechen von L bewirkt so ein Nachsprechen der Lautfolge bei $\mathrm{T}$, bis das richtige Wort imitiert ist. $\mathrm{L}$ fragt ihn dann, was denn ein Regenwurm sei (s9). Ihre Frage überschneidet sich aber noch mit der Antwort von T, der schon seine Aufmerksamkeit auf die nächste Abbildung gerichtet hat, die einen Hut darstellt. In (s8) benennt er diese mit Hüt und korrigiert sich dann zu (s10) Hut. Diese Selbstkorrektur, bei der er seine Äußerung 'repariert', kann laut GaRLIN (2000) nur dann vollzogen werden, wenn reflektiv das sprachliche Vorwissen miteinbezogen wird, das heißt der Ausdruck schon vorher wahrgenommen wurde. Hier liegt schon ein sprachliches Reflektieren im lexikalischen Bereich vor. L wiederholt

4 Der Terminus „Ausprobieren” wurde von REDDER und MARTENs (1983) eingeführt (GARLIN 2000). 
daraufhin in (s11) nochmals die Frage von (s9). T, der sich seiner Zweisprachigkeit bewusst ist, fragt L, ob sie eine Übersetzung des Wortes in das Portugiesische verlangt. Gefordert ist aber eine Erklärung auf Deutsch. Zugleich antworten beide Kinder mit ja. Tobias mit (s14) Ja, ich weiß, wobei $\mathrm{C}$ den Turn ergreift und mit (s15) Ja, wie ein Schlange die Lösung in Form des Handlungsmusters Beschreiben gibt. Hier zeigt sich, dass die Kinder bereits Musterwissen über die sprachlichen Handlungen Bestätigen und Beschreiben besitzen. Anschließend ergreift $\mathrm{C}$ wieder das Wort und beginnt mit einer Erzählung in (s18). Dieser Turn von C wird in Kapitel drei näher untersucht. Als C ihren Turn beendet hat, beginnt T in (s22) mit seinem Redebeitrag (Kapitel vier). Beide Äußerungen integrieren den Gegenstand Regenwurm, der vermutlich durch seine vorhergehende Benennung bei den Kindern ein Geflecht von Assoziationen aktiviert hat.

\section{B-3. Claudias Erzählen}

\section{B-3.1. Einleitungsphase}

\section{B-3.1.2. Erzählabsicht}

Um überhaupt Aussagen über die Erzählkompetenz der Kinder machen zu können bzw. um die Voraussetzungen für eine Erzählung zu analysieren, muss nach MENG et al. (1991) vor allem a) die Struktur der Äußerung sowie ihre Beschaffenheit beobachtet werden. Weitere wichtige Kriterien sind b) das Umfeld und die Situation, in der die Äußerung stattfindet, als auch c) deren Fortsetzung und Reaktionen auf sie.

Haben $\mathrm{C}$ und $\mathrm{T}$ das kommunikative Ziel, eine Erzählung zu planen? Das setzt unter anderem voraus, dass sie den Kommunikationstyp Erzäblen kennen. Es ist nun zu untersuchen, ob ihre kommunikativen Aktivitäten Aufschluss darüber geben, dass sie den Realisierungswunsch haben, eine Erzählung zu starten. Untersucht wird zuerst der Beitrag von C in Zeile (s18). Die Lehrerin initiiert das Frage-Antwort-Muster in einem Lehr-LernDiskurs, indem sie an Thomas die Frage stellt, was denn ein Regenwurm sei. Die beiden Kinder antworten gleichzeitig mit $j a$, wobei aber $\mathrm{C}$ mit ihrer Erklärung schneller ist und den Turn ergreift. Wie ein Schlange (s15) beschreibt 
sie. Daraufhin bewerten Thomas und die Lehrerin die Äußerung als richtig. Der Lösungsversuch von $\mathrm{C}$ ist gelungen und das Handlungsmuster der Lehrerfrage wurde positiv durchlaufen. C ergreift nach einer kurzen Pause wieder den Turn und kommt zu folgender Äußerung:

(s18) Aber, aber ich hab schon zwei gehabt.

C macht auf den ersten Blick eine Mitteilung über ein singulär vergangenes Ereignis, und zwar hatte sie schon einmal zwei Regenwürmer. Dies könnte der Anfang eines Erzählplanes sein, da dieses Ereignis für sie bedeutsam war, und es scheint der Wunsch zu bestehen, dies den anderen Kommunikationspartnern nun zu unterbreiten. Fraglich ist, ob $\mathrm{C}$ wirklich eine komplexere Ereignisdarstellung beginnen möchte oder ob sie nur eine Mitteilung macht. Der Kommunikationsmodus Mitteilung bei QuAstHOFF (1983) charakterisiert sich dadurch, dass die Turns jeweils nur einen Satz umfassen und es keine erkennbare Planung und Markierung gibt, dass eine längere Diskurseinheit folgen wird. Die Ereignisse werden vielmehr im Rahmen eines Turn-by-Turn-Talks vermittelt. Die Untersuchung des Fortgangs der Erzählung gibt Aufschluss darüber, ob C das Ereignis nur im Sinne einer Frage-Antwort-Sequenz äußert oder ob sie eine längere Diskurseinheit plant. Meng et al. (1991) unterscheidet fünf Typen von Möglichkeiten, die den Verlauf der weiteren Handlung entscheiden:

1) Der potentielle Erzähler liefert, ohne dass auf Seiten des Zuhörers spezielle Fragen gemacht wurden, spontan weitere Informationen über das von ihm bereits thematisierte Ereignis. Der Zuhörer bekundete lediglich ein allgemeines Interesse. Der Sprecher ist damit zum tatsächlichen Erzähler geworden.

2) Der prospektive Erzähler gibt unmittelbar nach der Eröffnungsäußerung weitere Informationen über das von ihm bereits thematisierte Ereignis, ohne dass der Zuhörer durch kommunikative Äußerungen Interesse bekundet hat. Auf diese Weise ist der potentielle Erzähler ebenfalls ein tatsächlicher Erzähler geworden. 
3) Obwohl der Zuhörer ein kommunikatives Interesse zeigt, liefert der Sprecher keine weiteren Informationen über das von ihm vorher schon Thematisierte.

4) Hier ist es dem potentiellen Erzähler nicht mehr möglich mit seiner Erzählung fortzufahren, da der Zuhörer nach einer möglichen Eröffnungsäußerung des Erzählers signalisiert, dass er die Erzähleinheit für abgeschlossen hält.

5) Nach der Eröffnungsäußerung kommt es zu keiner Zuhörerreaktion und der Sprecher versucht auch nicht, die Erzählung fortzusetzen.

(18) Aber, aber ich hab schon zwei gehabt. Aber eine hat ganz.ganz Wasser (....)

Bei der weiteren Betrachtung des Kommunikationsausschnitts von C sieht man, dass sie unmittelbar nach der Eröffnungsäußerung weitere Informationen liefert, ohne dass es dabei aber zu einem verbalisierten Interesse auf Seiten der Lehrerin oder ihres Bruders kommt. Die Fortführung erfolgt spontan, und somit ist 2) zutreffend. Denn damit offenbart sie ihre Erzählabsicht und geht über das Diskursmuster Mitteilung hinaus.

\section{B-3.1.3. Verteilung der Gesprächsrollen}

C bekommt die Erzählerrolle automatisch zugewiesen, indem sie, wie in 3.1.2 beschrieben, spontan weitere Informationen hinzufügt. Die Gesprächsrollen sind somit festgesetzt. Die Übernahme der Erzählerrolle geschieht bei ihr noch etwas impulsiv, ohne vorherige Prüfung, ob die Zuhörer aufnahmebereit sind.

\section{B-3.1.4. Einbettung in den laufenden Diskurs}

Erzählungen können nicht beliebig begonnen werden und können auch kein beliebiges Thema haben. Das liegt vor allem daran, dass der Kommunikationspartner in seiner Rezeptionsfähigkeit eingeschränkt ist. Auch 
erlauben die Handlungserwartungen ein 'Springen' von einem inhaltlichen Gegenstand zum anderen nicht (MENG et al. 1991). Es muss das Verhältnis des zuvor besprochenen Themas mit der neuen Kommunikationseinheit verglichen werden. Ein Turn kann dabei übernommen werden, wenn eine längere Gesprächspause herrscht oder wenn ein Thema abgeschlossen ist. Weicht ein neu eingeleitetes Thema völlig von der vorherigen Thematik ab, ohne dass der Themenwechsel explizit gemacht wurde, liegt ein 'Verstoß' gegen die sozialen Gesprächs-normen vor. Wie aus dem Transkript ersichtlich ist, knüpft die Erzählung von $C$ an die vorherige Thematik des Regenwurmes an. Die Einbettung dieser Erzähleinheit von $C$ entspricht also den geläufigen Kommunikationsnormen.

\section{B-3.1.5. Zusammenfassung}

Die wichtigsten Schritte wie Kontaktaufnahme (durch ihren Blickkontakt wurde ein gemeinsamer Handlungsraum hergestellt), Einbettung in den laufenden Diskurs sowie die Verständigung darüber, eine Erzählung zu beginnen, scheinen bei $\mathrm{C}$ gelungen zu sein. Ihre Erzählabsicht sowie die Verständigung darüber entwickelte sie relativ spontan, d.h. ohne Bekunden eines speziellen Interesses auf Seiten des Hörers. Die Einbettung fand zu einem geeigneten Zeitpunkt bei einer sinnvollen Themenfortführung statt. Die Erzählvoraussetzungen hat sie somit erfüllt.

\section{B-3.2. Hauptphase der Erzählung}

In der Hauptphase des Erzählens kommt es darauf an, für den Zuhörer primär die Verstehensvoraussetzungen zu schaffen und ihm somit eine Orientierung zu geben (s. 3.2.1). FLADER et al. (1980: 220) nennt diese Verstehensvoraussetzungen auch 'Versetzungsanweisungen'. Erst wenn der Bezugsrahmen des Geschehens geschaffen ist, beginnt der eigentliche Erzählvorgang (s. 3.3). In einem letzten Schritt kommt es zur Evaluation des Ereignisses, indem der Erzähler die eigene Sicht der Dinge darstellt (s. 3.4) und der Zuhörer nach Möglichkeit die persönliche Einstellung des Erzählers teilt (s. 3.5). 


\section{B-3.2.1. Orientierung}

Hierbei handelt es sich darum, einen gemeinsamen Vorstellungsraum zu schaffen. C muss den Zuhörer informieren, damit dieser die notwendigen Kenntnisse hat, um das Ereignis zu verstehen. Der Erzähler sollte erkennen, welche Zusatzorientierungen er dem Zuhörer mitgeben muss. Dabei gilt der Grundsatz, je mehr sich das Umfeld von Zuhörer und Sprecher ähnelt, desto weniger Hintergrundinformation muss gegeben werden (MENG et al. 1991). Zu den wichtigsten Informationen gehören: a) Ortsangaben, b) Zeitangaben, c) Personenangaben und Beziehung der Personen zueinander sowie deren Tätigkeiten, d) Angaben über Gegenstände, Objekte und deren Bedeutung in der Erzählung.

\section{B-3.2.2. Einführung von Orten}

Die Lebensbedingungen von T und $\mathrm{C}$ decken sich. Sie sind ein Geschwisterpaar und besuchen beide den Kindergarten. Die Anforderungen zur Orientierung untereinander sind also geringer, da sie ein umfangreiches gemeinsames Erfahrungswissen teilen. Allerdings unterscheidet sich der Lebensalltag der Lehrerin von dem der Kinder. Da die Lehrerin nur einmal in der Woche Kontakt zu den Kindern hat, kann man nicht davon ausgehen, dass sie genügend Hintergrundwissen hinsichtlich des Alltags der beiden Kinder hat. Die Orientierung muss in der Lehrer-Kind-Kommunikation in einem höheren Maße gegeben werden.

Die erste Voraussetzung nach lokaler Orientierung wird von $\mathrm{C}$ nicht gegeben. Ortsangaben sind aber nach der Konversationsmaxime der Quantität von GRICE (1975) nicht immer relevant, solange sie sich aus dem Kontext erschließen lassen. Trotzdem wäre es hier wichtig zu erfahren, wo das Ereignis stattgefunden hat. Handelt es sich um Regenwürmer, die zu Hause in einem Glas aufbewahrt wurden, oder befanden sich die Regenwürmer in freier Natur - vielleicht in einer Pfütze? C hat das gemeinsame Ortswissen zwischen ihr und der Lehrerin überschätzt. Die Ausgangssituation bleibt im Dunkeln. Hoffmann (1984) bemerkt dazu, dass es für Kinder ein großes Problem ist, sich in die Rolle und Perspektive des Gesprächspartners hineinzuversetzen und diese auch konstant miteinzubeziehen. 


\section{B-3.2.3. Zeitliche Einführung}

Beim Erzähltempus verwendet C das resultative Perfekt. Zur zeitlichen Orientierung gebraucht sie das Tempus des Verbs und macht damit deutlich, dass das Ereignis sich von der Zeit der aktuellen Kommunikationssituation unterscheidet.

\section{B-3.2.4. Einführung von Personen/Objekten}

In narrativen Diskursen geht es immer um Vorgänge, die sich auf Personen (Aktanten) beziehen, oder um von Personen ausgeführte Handlungen. Oder aber um Ereignisse, in denen eine Veränderungen an Objekten den Mittelpunkt bilden. Eine wichtige Voraussetzung ist es somit, dass der Erzähler die Identität der Personen sicherstellt, sodass er im Verlauf der weiteren Erzählung ohne Verständnisschwierigkeiten auf sie zurückgreifen kann. Wenn die Aktanten dem Hörer nicht bekannt sind, werden diese bei der Ersterwähnung indefinit eingeführt. Damit wird ihre Existenz erstmals angedeutet (BOUEKE et al. 1995). Als weitere sprachliche Ausdrücke bieten sich indefinite Nominalphrasen meist in Verbindung mit einem Appellativum an. Hat der Zuhörer das nötige Hintergrundwissen, müssen sprachliche Ausdrücke verwendet werden, die den Zuhörer hinsichtlich seines Identifizierungswissen aufmerksam machen. Definite Ausdrücke, Eigennamen oder deiktische Mittel stehen dem Sprecher dabei zur Verfügung. Wie schafft es C, Personen und Gegenstände einzuführen und weiteres, auf diese bezogenes Wissen, zu reaktivieren? Sie bezieht sich mit ihrer Aussage (s18) Aber, aber ich hab schon zwei gehabt auf den eingeführten Gesprächsgegenstand (Regenwurm) in Zeile (s13). Dabei verwendet sie das Zahlen-adjektiv zwei, das zur Verständnissicherung völlig ausreicht. Der Zuhörer kann sich noch gut auf sein Identifizierungswissen von der vorausgehenden Äußerung (s13) stützen. Es gelingt ihr somit, den Regenwurm wieder in die gemeinsame Aufmerksamkeit zu rücken. Die Orientierung auf den Regenwurm ist ihr gelungen. C führt sich als Person mit $i c h$ ein. Sie greift auf diesen sprecherdeiktischen Ausdruck zurück, da sie sich im gemeinsamen Wahrnehmungsraum befindet. Durch die lexikalische Verbform hab gebabt drückt sie die Beziehung zu dem Regenwurm aus. 


\section{B-3.2.5. Zusammenfassung}

Die Äußerungen von $\mathrm{C}$ zeigen, dass sie ein gewisses Orientierungswissen hat. Zur zeitlichen Orientierung benutzt sie das Perfekt. Für die Einführung von Personen sind ihr sprecherdeiktische Elemente bekannt. Allerdings gibt es noch keine selbstständigen sprachlichen Handlungen (Sätze), die Orientierungswissen vermitteln.

\section{B-3.3. Darstellung der Ereignisfolge}

Zur wichtigsten Phase einer Erzählung gehört die Ereignisfolge. Die Ereignisfolge zeichnet sich durch Geschlossenheit aus. Als Voraussetzung für Geschlossenheit dient die kontinuierliche Identität von Personen und Gegenständen, die an der Ereignisfolge beteiligt sind. Mit der Einführung von Gegenständen/Personen haben wir uns in Kapitel 3.2.4 beschäftigt. Im weiteren Verlauf der Darstellung geht es nur darum, diese im Fokus zu behalten. Die Beibehaltung geschieht mittels pronominaler Ausdrücke, die semantisch nicht isoliert vom Kontext interpretiert werden können, da sie sich auf das bereits zuvor Erwähnte beziehen. C kennzeichnet in ihrer Ereignisdarstellung die kontinuierliche Identität des eingeführten 'Gegenstandes' Regenwurm (s13) durch verschiedene sprachliche Mittel. Mit dem Zahlenadjektiv ${ }^{5}$ eine soll der Fokus auf einen der beiden Regenwürmer gelenkt werden. Sie benutzt dabei das falsche Genus; die feminine Form. Es könnte sein, dass sie sich auf die Schlange in (s15) bezieht, denn schon in der nächsten Folgeerwähnung verwendet sie das richtige Genus. In ihrer Verweiskette verwendet sie nun den definiten Artikel (und der hat schon tot), damit die Aufmerksamkeit auf dem Regenwurm beibehalten wird, der 'im Hochwasser' ist. Bei der letzten Handlungskette (und vom vom Thomas hat sich verloren) fehlt die Referenz. Allerdings kann man aus dem Gebrauch der Präposition und dem Appellativum (Thomas) schließen, dass mit dem verlorenen Regenwurm der andere Regenwurm gemeint ist.

Laut Helbig \& Buscha (1987) handelt es sich um ein Zahlenadjektiv im Sinne der eine und der andere, die keine genaue Reihenfolge angeben. 


\section{B-3.3.1. Zeitliche Beziehung}

Die zeitliche Markierung kann durch Tempusformen oder durch die explizite Erwähnung mittels lexikalischer Mittel (erst, später usw.) erfolgen (MENG et al. 1991). C macht keinen Gebrauch von lexikalischen Mitteln, sondern verwendet zur Kennzeichnung der Ereignisfolge die temporale Verbform des Perfekts (s. auch Eröffnungsäußerung). Allerdings gelingt ihr die Bildung des Perfekts nicht immer. Schon in der zweiten Zeile in (s18) lässt sie das Partizip weg. Im angeschlossenen Hauptsatz benutzt sie anstatt des Partizips gestorben das Adjektiv tot zusammen mit dem Hilfsverb hat. Im letzten Satz verwendet sie schon das reflexive Verb sich verlieren, verbalisiert aber die falsche partizipiale Form dazu (verlieren). Obwohl ihr die Bildung des Perfekts nicht immer gelingt, kann man aber die Binnenstruktur der Geschichte nachvollziehen.

\section{B-3.3.2. Zusammenfassung}

C erzählt zwar wesentliche Teile der Ereignisfolge, insgesamt bleibt aber die Erzählung bruchstückhaft und die einzelnen Aspekte werden zu wenig verbalisiert. Die Rekonstruktion der Geschichte bleibt in vielen Teilen offen. Es ist nicht klar, warum der Regenwurm im Wasser war und ob er dann auch wirklich ertrunken ist. Unklar ist auch, wo und wie der Regenwurm von Thomas verloren gegangen ist. Insgesamt ist die Geschichte zu wenig detailliert. Der Mittelpunkt von Cs Erzählung bildet eher das Resultat. Vorund Nachgeschichte werden vernachlässigt. Allerdings zeigt der Gebrauch von indefiniten Nominalphrasen und deren Übergang zu definiten Nominalphrasen, dass C Fragmente der Fokussierung beherrscht.

\section{B-3.4. Bewertung}

Ein wichtiges Merkmal einer Erzählung ist die Bewertung. Der Erzähler muss seine persönliche Einstellung hinsichtlich des Ereignisses vermitteln können. Dies unterscheidet das Handlungsmuster Erzählen von anderen Ereignisdarstellungen. Dabei kann die eigene Einstellung laut Meng et al. (1991) schon während der Ereignisdarstellung kenntlich gemacht werden und muss nicht erst in der Abschlussphase gekennzeichnet werden. MENG 
et al. (a.a.O.) unterscheiden Bewertungen verbaler und nonverbaler Art. Bewertungen verbaler Art können lexikalisch, thematisch oder in einem Akzentuierungsverfahren geäußert werden (Meng et al. 1991: 87). C stellt weder während der Ereignisdarstellung noch am Ende der Darstellung ihre Sicht der Dinge verbal dar. Allerdings erzählt sie die Geschichte, nach der Stimmcharakteristik zu deuten, in einem ernsten Tonfall. Daraus kann man schließen, dass das Ereignis eher traurig für sie war. Doch ist diese Schlussfolgerung sehr vage. Während der Erzählung verwendet sie außerdem viel Gestik und Mimik, was auch auf eine besonders starke emotionale Beteiligung hindeuten kann. Ihre Sichtweise in Bezug auf das erzählte Ereignis ist nicht eindeutig erkennbar. Bezüglich des Motivs, das ihrer Erzählabsicht zugrunde liegt, kann man sagen, dass sie wahrscheinlich das Ereignis als für die Zuhörer interessant und informativ einstuft und deshalb ihre Darstellungsabsicht entwickelt, um die anderen daran teilhaben zu lassen.

\section{B-3.5. Abschlusshandlung des Zuhörers}

Nachdem die Erzählung unerwartet beendet wird, übernimmt die Lehrerin den Turn. Mit dem betonten Fragepronomen (s19) was, mit dem sie die Unerhörtheit dieser Geschichte verdeutlicht, vermittelt sie C, dass die Geschichte eine außergewöhnliche ist. Gleichzeitig erzählt L die Geschichte nach. Diese Nacherzählung kann zur Verständnissicherung dienen, da die Darstellung von $C$ sehr reduziert war und es einige Verstehensprobleme gab. L versucht, die Darstellung zu ergänzen und vermittelt zugleich das vollständige sprachliche Muster an C. L formuliert die Nacherzählung als eine Frage, was darauf hindeuten könnte, dass sie $\mathrm{C}$ um eine Bestätigung bittet oder indirekt $\mathrm{C}$ auffordert, die Erzählung fortzusetzen. Die Entscheidungsfrage wird von $\mathrm{C}$ mit der Interjektion (s20) hm beantwortet, die hier eine Zustimmung, eine Konvergenz ausdrückt. Die Bejahung erfolgte schnell und unkritisch, was zur Annahme führt, dass sie sich des Wahrheitsgehaltes, die eine Erzählung fordert, nicht bewusst ist. Sie scheint ein erinnerungskritisches Reflektieren nicht für notwendig zu halten. L signalisiert nochmals ihr Verstehen der Darstellung von C ebenfalls mit der Höreräußerung (s21) hm (in Aussageintonation) und markiert den Abschluss der Kommunikationseinheit durch ihre Äußerung in (s21): Das hört sich ja gut an. O.K. 
Auffällig ist, dass L die Nachformulierung in einem belustigten Tonfall vollzieht und somit ihre Einstellung und Bewertung hinsichtlich der Erzählung manifestiert. Aufgrund der mimischen Aktivität Cs (lächelnder Gesichtsausdruck) scheint auch sie die Sichtweise von L zu übernehmen.

\section{B-4. Tobias' Erzählen}

\section{B-4.1. Transkriptbeschreibung}

Tobias, der sich als Zuhörer nicht beteiligt hat, ergreift in Zeile (s22) den Turn. Zur Gewinnung des Rederechts beginnt er mit der formelhaften Wendung: (s22) Weißt du schon, was ich gemacht habe? Damit kündigt er unmittelbar seine Erzählabsicht an und befragt auch gleichzeitig das Hörerwissen (Weißst du schon...). Der kommunikative Kontakt ist geknüpft. L reagiert auf das Erzählangebot und bekundet ihr Interesse in (s23) mit: Was denn? Die Verständigung darüber, dass T im Kommunikationstyp Eræählen kommunizieren will, scheint gelungen zu sein. Laut der verschiedenen Typen von Handlungszusammenhängen zur Bestimmung der kommunikativen Aktivitäten (s. Abschnitt 3.1.2) zwischen potentiellem Erzähler und Zuhörer, trifft Typ $1 \mathrm{zu}$. Der Grund für die Inanspruchnahme des Rederechts könnte die Forderung nach Gleichberechtigung sein. T hat registriert, dass $\mathrm{C}$ die Aufmerksamkeit der Lehrerin genoss, und bemüht sich nun auch um deren Aufmerksamkeit. T beginnt seinen Turn, nachdem die Kommunikationseinheit von $\mathrm{C}$ sichtbar abgeschlossen ist. Die Bedingungen zur Einbettung in die laufende Kommunikation sind von ihm erfüllt. Es zeigt sich hier, dass er das Turn-Taking beherrscht.

Mit der einleitenden zeitlichen Orientierung (s24) Ich hab schon/Ich war schon einmal (...) führt T seinen Turn fort. Er vollzieht die zeitliche Einordnung mit Hilfe des Verbs im Präteritum. Diese Erzähleinheit weist unter anderem Versatzstücke aus Märchen und anderen Kindergeschichten auf, die ihm wohl als strukturelles Vorbild dienen. Er gibt an, dass er mit dem Radl gefahren ist und gibt dem Zuhörer damit eine wichtige Verstehensvoraussetzung zur räumlichen Orientierung. Mit der Formulierung: (s24) Weißt du was ich gesehen habe? gebraucht er ein erzählerisches Mittel zur 
Spannungssteigerung. Das Resultat ist: (s24) Eine Schnecke, eine Schnecke hoch bei eine, eine.

Wie in Kapitel 3.2.4 erklärt wurde, erfolgt die Orientierung über den Gegenstand durch seine Ersterwähnung. T verwendet hier das Grundmuster der Einführung von Gegenständen; den indefiniten Artikel. Zur weiteren Fortführung des Ereignisses fehlen ihm aber die Worte. Die Schwester versucht ihm mit dem Symbolfeldausdruck ${ }^{6}$ (s25) Baum zu helfen. Das ist aber nicht das gesuchte Wort. T: (s26) Wie eine Schnecke, wie heißt die? T ist sich seines Wissensdefizits bewusst und formuliert dazu das Handlungsmuster Frage. Er hat eine genaue Vorstellung von dem Tier, das er sucht. Es sieht so aus wie eine Schnecke. L vermutet ein Schneckenhaus (s28). T gibt nochmals eine detailliertere Beschreibung. (s39) Äh, nein. Die war riesig. Wie heißt des? $?^{7}$ Und in Zeile (s31) Die so wie $n$ Schlange. Vermutlich meint er eine spezielle Schneckenart. Es könnte sich dabei um eine Nacktschnecke ${ }^{8}$ handeln. Die Vermutung von L, dass es sich um einen Wurm handelt, kommt dem gesuchten Begriff schon näher. (s33) Ja, ein Wurm. C kommt wieder in Zeile (s36) auf den Regenwurm zurück. Mit Regenwurm scheint er dann zufrieden zu sein. (s37) Ah ja, ein Regenwurm. Dann versucht er die Geschichte noch einmal zu erzählen, aber der Neuansatz mit seiner Umstrukturierung macht ihm Schwierigkeiten. (s37) Ich hab ein Wurm/, äh ein Regenwurm/äh der Schnecke war hoch auf die Regen/ auf die Regenwurm. Hier möchte er wahrscheinlich erklären, dass der Regenwurm unter der Schnecke war. Trotz der anakoluthischen ${ }^{9}$ Selbstkorrektur gelingt ihm der Ansatz nicht und er beginnt wieder, wie bei seinem ersten Erzählversuch, mit der Schnecke, die auf dem Regenwurm saß. T beendet hier vorerst seinen Turn. L reagiert auf die Erzählung von T mit einer erstaunt-ungläubigen Entscheidungsfrage, die ihre Vermutung bestätigen soll. (s38) Ab, die Schnecke war auf dem Regenwurm drauf? Durch das Handlungsmuster Frage bittet sie gleichzeitig auch um eine Fortsetzung der Darstellung oder zumindest um eine

$6 \quad$ Symbolfeldausdrücke bezeichnen und benennen Gegenstände und Sachverhalte.

7 Umgangssprachliches Demonstrativpronomen des anstatt das.

8 Die Mutter erzählte, dass das Wort Nacktschnecke auf Portugiesisch bekannt ist (portugiesisch: lesma).

$9 \quad$ Anakoluth $=$ folgewidrige Fortsetzung einer angefangenen Satzkorrektur. 
Bewertung. Erst jetzt erzählt T den eigentlichen Höhepunkt der Geschichte und das Resultat, nachdem er mit einem Nicken die Vermutung von L bestätigte. (s41) Und und sie hat sie getötet und sie essen sie. Vermutlich verwendet er hier neben dem Perfekt das Präsens zur Hervorhebung und Vergegenwärtigung des Ereignisses. Es handelt sich wohl kaum um ein Präsens, das sich auf allgemein Gültiges bezieht oder zur generellen Aussage formuliert wird, obwohl die falsche Deklination des Personalpronomens sie statt ibn und die falsche Verbkonjugation essen statt isst zu dieser Annahme führen kann. L bezweifelt die Glaubwürdigkeit der Aussage. (s42) Ehrlich? Die essen die? Schnecken essen Regenwürmer? Mittels objektdeiktischer ${ }^{10}$ Ausdrücke wiederholt sie die Aussage von $\mathrm{T}$ und verstärkt ihre Zweifel, indem sie noch einmal mit Hilfe der Symbolfeldausdrücke Tobias' Aussage wiederholt. HofFmann (1984) weist daraufhin, dass das Kriterium der Wahrheit für Kinder im Vorschulalter keine große Rolle spielt und Erzählungen, die sich explizit auf eigene Erfahrungen beziehen, oftmals eine Vielzahl fiktionaler Elemente enthalten. T, der jetzt etwas verunsichert ist, beteuert noch zweimal, dass er dies gesehen hat. (s44) Ja ((1s)), ja. ((unsicher)) Sie hat gemacht. Ehrlich. Ich hab sie gesehen hoch bei Schnecke. Und sie essen (s45) L: Das kann natürlich sein. (s46) T: Sie hat sie schon, sie hat sie schon getötet. Gegen die Haltung des Anzweifelns der Lehrerin und um den Wahrheitsgehalt zu verdeutlichen, betont er nochmals, dass er dies selbst gesehen hat und verstärkt dies mit einem umgangssprachlichen ehrlich, im Sinne von ganz bestimmt. Auch unterstreicht die zweimalige Verwendung der Modalpartikel schon (betont), den Rechtfertigungscharakter der Äußerung. L beendet die Kommunikationseinheit mit: (s47) Getötet. Ja, so was.

\section{B-4.2. Zusammenfassung}

Zur Herstellung des kommunikativen Kontakts verwendet $\mathrm{T}$ schon die bekannte Einleitungsformel für Erzählungen. T zeigt sich in der Lage, seine Kommunikationseinheit zu einem geeigneten Zeitpunkt zu platzieren. Seine Themenwahl passt in den Gesamtzusammenhang der Gesprächs-

10 Objektdeiktische Ausdrücke verweisen auf Objekte im Verweisraum (dazu zählen Demonstrativpronomen wie dieser, jener, das). 
situation. Die funktionsgerechte Verwendung von indefiniten Formen bei der Ersterwähnung von Personen/Gegenständen ist gelungen. Zur Fokussierung auf fortlaufende Gegenstände verwendet er objektdeiktische Prozeduren (s26; s29, s31). Er beherrscht auch schon die anaphorischen ${ }^{11}$ Prozeduren wie in (s41) Und sie hat sie getötet und sie essen sie, indem er mittels Personalpronomen einen Rückbezug auf die Subjekte des vorhergehenden Satzes herstellt. Da er aber sprachliche Schwierigkeiten bei der Verwendung der richtigen Artikel und Präpositionen hat, ist die referentielle Eindeutigkeit nicht immer klar. Ansatzweise gibt T auch schon eine satzartige lokale Orientierung. Ihm gelingt auch der standardsprachliche Wechsel zwischen den Tempora Präteritum und Präsens zur Markierung der zeitlichen Orientierung. Allerdings wird die chronologische Darstellung immer wieder durch die Suche nach dem Wort Regenwurm unterbrochen. Hier macht sich sein fehlendes Vokabular bemerkbar. Erst durch die Fortsetzungserwartung der Lehrerin kommt es dann auch zur Erzählung eines Höhepunktes und eines Resultats. Eine Bewertung jedoch bleibt aus. Wie aber aus der Reaktion des erwachsenen Zuhörers hervorgeht, erfüllt $\mathrm{T}$ die zweite wichtige Kategorie des Erzählens; die 'Kategorie der Ungewöhnlichkeit'. Der Wahrheitsgehalt des Ereignisses scheint der Lehrerin aber eher zweifelhaft zu sein (s38, s42). Doch kann das darauf zurückgeführt werden, dass mit Regenwurm eine falsche Benennung vorliegt. Würde es sich um eine Schnecke handeln, die sich auf einer Nacktschnecke befindet, entspräche dies durchaus der Realität. Auffällig ist auch, dass L bei T in einem größeren Maß ihre Verständnisschwierigkeiten signalisiert, als sie dies bei $C$ tut. Sie schätzt wahrscheinlich die Erzählkompetenz bei T höher ein. Hinsichtlich der Zuhöreraktivitäten bei $\mathrm{C}$ ist zu bemerken, dass sie sich während des Diskurses von $\mathrm{T}$ nicht in der Position des primären Adressaten befindet. Sie gibt außer in Zeile (s43) keine Zuhöreräußerungen. Auch diese Zuhörerbeteiligung ist nur durch Vermittlung von L zustande gekommen, indem sie mit Blickkontakt die Frage in (s42) auch an C stellt. C ist weniger als Zuhörer tätig und zeichnet sich vielmehr als helfende Teilnehmerin aus. Auffällig ist auch, dass die Kinder in der Position des Erzählers sich ausschließlich dem erwachsenen

11 Die Anapher ist eine sprachliche Einheit, die auf etwas Vorausgegangenes verweist. 
Zuhörer zuwenden. Ein Grund hierfür könnte sein, dass sie sich die Geschichte untereinander schon erzählt haben, und so die Geschichte beim Anderen als bekannt voraussetzen. Es ist aber anzunehmen, dass sie den erwachsenen Zuhörer als kompetenteren Gesprächspartner bevorzugen, da Kinder in der Zuhörerrolle kaum aktiv sind.

\section{B-5. Schlussbemerkung}

Insgesamt ist die Erzählfähigkeit, trotz der unterbrochenen Darstellung, bei T weiter entwickelt als bei C. Dies lässt sich darauf zurückführen, dass T älter und außerdem institutionell 'vorgeprägt' ist. Das Musterwissen über Erzählungen ist aber bei beiden auf jeden Fall in Ansätzen vorhanden. Es ist davon auszugehen, dass das Handlungsmuster Erzä̆blen im Portugiesischen ähnlich realisiert wird und die Kinder so bei ihrer Ankunft in Deutschland schon über das Musterwissen Eræä̈blen verfügten. Somit sind sie den deutschen Kindern vielleicht in dieser Hinsicht nicht unterlegen, (eine parallele Analyse mit deutschen Kindern wäre hierbei hilfreich) sondern der Grund für ein nicht erfolgreich realisiertes Muster liegt eher an einem unzureichenden sprachlichen Ausdruckswissen. Eine Erzählkompetenz im Deutschen sagt noch nichts über das Portugiesische aus, weil die sprachlichen Mittel zur Vollziehung des Musters bei den beiden Sprachen unterschiedlich sind.

In Anbetracht dieser Feststellung halte ich eine Sprachförderung in der Zweitsprache für sehr sinnvoll, denn die Kinder brauchen Hilfe beim Erlernen von Musterwissen (andere Sprachen können andere Handlungsmuster aufweisen) und von den sprachlichen Mitteln, um diese realisieren zu können. Das Konzept von KIKUS sieht unter anderem vor im Sinne des interkulturellen Lernens auch die Erstsprache miteinzubeziehen. Das heißt, die Kinder sollen nicht ihre Erstsprache 'ausschalten', sondern werden aufgefordert, sprachliche Ausdrücke auch in dieser zu gebrauchen. So werden die Kinder darauf hingewiesen, dass es zwei sprachliche Möglichkeiten gibt, um Objekte oder Handlungen zu verbalisieren. Nicht zuletzt entspricht dieses Konzept den internationalen Entwicklungen und Ansprüchen einer im Bildungswesen verankerten Mehrsprachigkeit und trägt dem politischen Ziel der sozialen Integration von Kindern mit Migrationshintergrund Rechnung. 


\section{Literaturverzeichnis}

Boueke, D. / Schülein, F. / Büscher, H et al. Wie Kinder erzählen. Untersuchungen zur Erzähltheorie und zur Entwicklung narrativer Fähigkeiten. München, Fink 1995.

BüHLER, K. Die geistige Entwicklung des Kindes. Jena, Fischer 1922.

Eнtich, K. "Der Alltag des Erzählens". In: Eнtıch, K. (Hg.): Eræä̆blen im Alltag, Frankfurt, Suhrkamp 1980, 11-25.

EHLIch, K. “Alltägliches Erzählen”. In: SANDERs, W. / Wegenast, K. (Hg.): Erzäblen für Kinder - Eræählen von Gott, Stuttgart, Kohlhammer 1983a, 128-151.

EHLich, K. "Sprachanalyse”. In: Enzyklopädie Erziehungswissenschaften. Bd.2: Methoden der Erziehungs- und Bildungsforschung, Stuttgart, Klett-Cotta 1983b, 526-538.

Ehlich, K. „Zum Textbegriff”. In: Rothkegel, A. / SAndig, B. (Hg.): Text, Textsorten, Semantik, Hamburg 1984a, 9-25.

EHLICH, K. Erzählen in der Schule. Tübingen, Narr, 1984b.

Eнtich, K. "Funktionale Pragmatik: Terme, Themen und Methoden". In: Deutschunterricht in Japan H.4/1999, 4-24.

Ehlich, K. / Rehbein J. "Halbinterpretative Arbeitstranskriptionen (HIAT)". In: Linguistische Berichte 45-46/1976, 21-46.

Ehlich, K. / Rehbein, J. "Sprachliche Handlungsmuster". In: Soeffner, H.-G. (Hg.): Interpretative Verfahren in den Sozial- und Textwissenschaften, Stuttgart 1979, 243-274.

Flader, D. / GieseCKE, M. "Erzählen im psychoanalytischen Erstinterview - eine Fallstudie". In: Ehlich, K. (Hg.): Erzäblen im Alltag, Frankfurt, Suhrkamp 1980, 209-263.

GARLIN, E. “Erstsprach(en)erwerb: Vom „Ausprobieren” zum sprachlichen Handeln". In: RedDer, A. (Hg.): Diskursanalysen in praktischer Absicht. Osnabrücker Beiträge zur Sprachtheorie 49/1994, 82-105.

GARLIN, E. Bilingualer Erstspracherwerb. Sprachliches Handeln - SprachprobierenSprachreflexion. München, Verlag für Sprache und Sprachen 2000.

Grice, H.-P. "Logic and Conversation”. In: Davidson, D. / Harman, G. (Hg.): The logic of Grammar, Encino, Calif. 1975, 64-75. 
Helbig, G. / Buscha, J. Deutsche Grammatik. Ein Handbuch für den Ausländerunterricht. Leipzig, VEB Verlag 1987.

Hoffmann, L. "Zur Ausbildung der Erzählkompetenz: eine methodische Perspektive”. In: EHLIcH, K. (Hg.): Erzä̈hlen in der Schule, Tübingen, Narr 1984, 202-222.

Meng, K. / Kraft, B. / Nitsche, U. Kommunikation im Kindergarten. Studien zur Aneignung kommunikativer Kompetenz. Berlin, Akademie 1991.

Quasthoff, U.-M. "Kindliches Erzählen. Zum Zusammenhang von erzählendem Diskursmuster und Zuhöreraktivitäten”. In: Boueke, D. / KieIN, W. (Hg.): Untersuchungen zur Dialogfähigkeit von Kindern, Tübingen; Narr 1983, 45-75.

Redder, A. / Martens, K. "Modalverben ausprobieren - wie Kinder mit Modalverben handeln". In: BoueKe, D. / KLEIN, W. (Hg.): Untersuchung von Dialogfähigkeit bei Kindern, Frankfurt, Lang 1983, 163- 183.

ReHbein, J. "Sequentielles Erzählen- Erzählstrukturen von Immigranten bei Sozialberatungen in England”. In: EHLich, K. (Hg.): Erzählen im Alltag, Frankfurt, Suhrkamp 1980, 64-109.

Romaine, S. Bilingualism. Oxford / New York, Blackwell, 1989.

Wagner, K.-R. / SteinsRÄter, C. "Individuelle Profile beim Erzählerwerb". In: Ehlich, K. / WagneR, K.-R. (Hg.): Erzähl-Erwerb. Frankfurt, Lang 1989, 49-62.

Weber, U. Instruktionsverhalten und Sprechhandlungsfähigkeit. Tübingen, Niemeyer 1982.

Zifonun, G. / Hoffmann, L. / Strecker, B. et al. (Hg.): Grammatik der deutschen Sprache. Bd. 1 Sprache und Illokution. Berlin, de Gruyter 1997.

\section{Anhang}

Transkript: Freies Erzählen

Aufnahmedauer: 2 Minuten

Siglen: L Lehrerin, T Tobias, C Claudia 


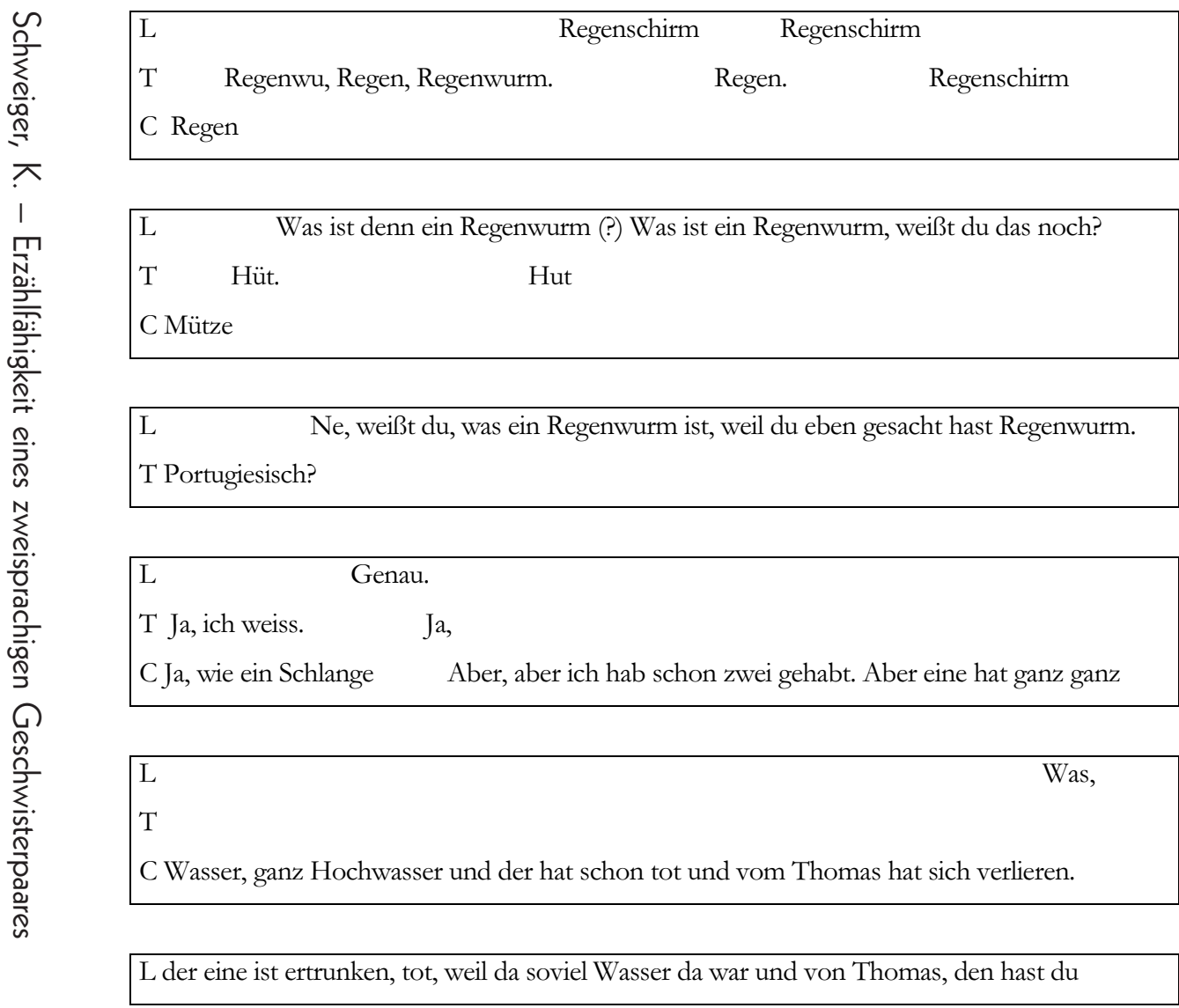

\begin{tabular}{|lrrr|}
\hline L verloren? $\mathrm{Hm}$ & Hört sich ja gut an. O.k. & Was denn? \\
$\mathrm{T}$ & & Weißt du was, was ich gemacht habe? \\
$\mathrm{C}$ & $\mathrm{Hm}$ & \\
\hline
\end{tabular}

L
T Ich war schon/Ich war schon einmal, einmal ((zögernd)) einmal äh mit die Ra/mit meinem

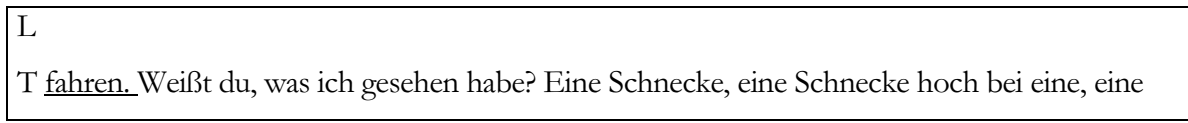

\begin{tabular}{|l|l|}
$\mathrm{L}$ & \\
$\mathrm{T}((1 \mathrm{~s}))$ \\
$\mathrm{C}$ & Baum.
\end{tabular}




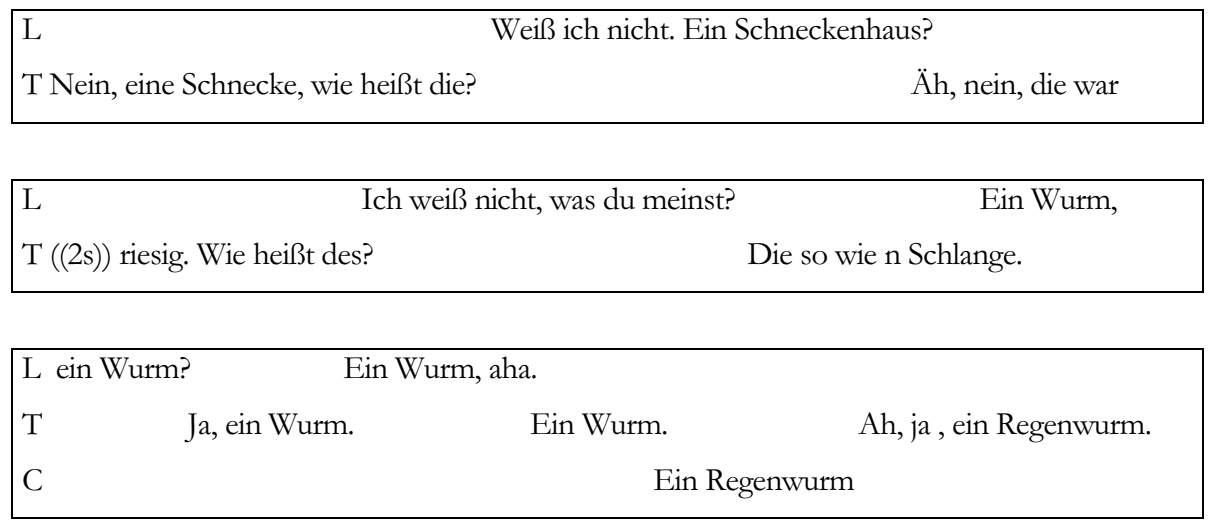

L
T Ich hab/ ein Wurm, äh ein Regenwurm/äh der Schnecke war hoch auf die Regen/auf die

\begin{tabular}{|lll|}
\hline $\mathrm{L}$ & Ah, die Schnecke war auf dem Regenwurm drauf? & Ehrlich? \\
T Regenwurm. & $($ (nickt) $)$ & Und sie hat \\
\hline
\end{tabular}

\begin{tabular}{|lc|}
\hline L & Ehrlich? Die essen die? Schnecken essen Regenwürmer? \\
T sie getötet und sie essen sie. & Weiß \\
C & We \\
\hline
\end{tabular}

\begin{tabular}{|l|}
\hline $\mathrm{L}$ \\
$\mathrm{T} \quad \mathrm{Ja},((1 \mathrm{~s}))$, ja ((unsicher)), sie hat gemacht, ehrlich Ich habe sie gesehen hoch bei \\
C ich nicht!
\end{tabular}

\begin{tabular}{|l|}
\hline $\mathrm{L}$ \\
$\mathrm{T}$ bei Schnecke. Und sie essen.
\end{tabular}

\begin{tabular}{|l|}
\hline $\mathrm{L} \quad$ Getötet. Ja, so was. \\
T getötet.
\end{tabular}

